\title{
Limbic Encephalitis Associated with
} Voltage-Gated Potassium Channel-Complex Antibodies: Patient Report and Literature Review

\author{
David S. Younger \\ Department of Neurology, New York University School of Medicine, and the College of Global Public Health, \\ New York University, New York, USA \\ Email: David.younger@nyumc.org
}

How to cite this paper: Younger, D.S. (2017) Limbic Encephalitis Associated with Voltage-Gated Potassium Channel-Complex Antibodies: Patient Report and Literature Review. World Journal of Neuroscience, 7, 19-31.

https://doi.org/10.4236/wjns.2017.71002

Received: December 1, 2016

Accepted: December 20, 2016

Published: December 23, 2016

Copyright $\odot 2017$ by authors and Scientific Research Publishing Inc. This work is licensed under the Creative Commons Attribution International License (CC BY 4.0).

http://creativecommons.org/licenses/by/4.0/

\begin{abstract}
Background: The family of disorders associated with antibodies against neuronal surface antigens is a rapidly expanding category of autoimmune neurologic disease. Methods: Clinical and laboratory findings of a patient with voltage-gated Kv1 potassium channel (VGKC)-complex autoantibodies are reported and the literature is reviewed. Results: New-onset faciobrachial dystonic seizures, distal large and painful small fiber peripheral neuropathy, dysautonomia, cognitive and memory disturbances were associated with hypometabolism of the left temporal lobe without evidence of systemic malignancy in whole-body positron emission tomography with 2-deoxy-2-[fluorine-18] fluoro-D-glucose integrated with computed tomography. Brain MRI and cerebrospinal fluid were normal with absent intrathecal VGKC-complex antibodies consistent with extrathecal production. Epidermal nerve fiber studies confirmed small fiber neuropathy in association with abnormal autonomic laboratory testing. Discussion: Neurologic involvement associated with VGKC complex autoimmunity is a precisely delineated disorder involving the central, peripheral, and autonomic nervous system.
\end{abstract}

\section{Keywords}

Autoimmune, Encephalitis, Neuronal Surface Antigens

\section{Introduction}

Autoantibodies against the voltage-gated Kv1 potassium channel-(VGKC)complex detected by radio immunoprecipitation assay (RIA) in the sera of patients with autoimmune encephalitis do not bind to VGKC-complex channel 
proteins, but instead bind to synaptic and axonal neuronal proteins that coprecipitate with detergent-solubilized VGKC [1] [2]. Attention has been directed toward identifying the principal autoantigens in the VGKC-complex and an expanding spectrum of corresponding phenotypes. The principal autoantigens most commonly defined are leucine-rich glioma-inactivated protein 1 (LGI1) in the central nervous system (CNS) and contactin-associated protein-like2 (CASPR2) which associates with transiently-expressed axonal glycoprotein (TAG1), post synaptic density Protein-Drosophila disc large tumor suppressor-zonula occludens-1 protein (PDZ), and the ankyrin-spectrin protein in both the peripheral nervous system (PNS) and CNS [1] [2]. There is a diversity and overlap of neurological phenotypes that relate to VGKC-Complex-IgG values in the serum and CSF and Ig-subtype specificity. The commonest presentation of VGKCcomplex autoantibodies is LE in the CNS and neuromyotonia or Morvan syndrome in the PNS [3]. We present the clinical and laboratory findings of a patient that manifested PNS, CNS and autonomic nervous system (ANS) involvement.

\section{Patient Report}

A 68-year-old woman developed postural lightheadedness and palpitations in February 2015, followed four months later in June 2015 by neuropathic pain, numbness, tingling and weakness of the arms and legs. In March 2016 she developed unprovoked episodes of disturbed vocalizations, facial grimacing, left head turning, neck stiffness, and left arm posturing lasting a few seconds, occurring up to a dozen times per day. Prior to evaluation at New York University Langone Medical Center in June 2016, she noted progressive memory loss and personality change. Past medical history was not contributory. She was allergic to clindamycin, penicillin, and Levaquin. She took no medications. Family history was not contributory. General examination in June 2016 was normal. Neurological examination showed stocking sensory loss, Romberg sign, tandem imbalance, distal leg weakness, and hyporeflexia with intact cranial nerves and mental status.

A standard 21-channel awake and drowsy electroencephalogram (EEG) preformed in January 2016, early in the course of her illness was normal. Electrodiagnostic (EDX) studies of the legs performed in June 2016 under standard conditions of temperature using a Nicolet Viking Select Viasys ${ }^{\oplus}$ system showed distal demyelinating and secondary axonal changes in the legs. The left tibial motor distal motor latency (DL) was 7.8 millisecond (ms) (normal $6.8 \mathrm{~ms}$ ), with a compound muscle action potential (CMAP) amplitude of 1.9 millivolts (mV) (normal $3 \mathrm{mV}$ ), abnormal temporal dispersion, and motor nerve conduction velocity (MNCV) velocity of 36 meters per second $(\mathrm{m} / \mathrm{s})$ in the popliteal-to-ankle segments; and a right tibial MNC velocity of $35 \mathrm{~m} / \mathrm{s}$, also with abnormal temporal dispersion but normal DL. Bilateral fibular EDX studies were normal. Bilateral superficial fibular sensory and right sural responses were not obtained, however the right sural sensory nerve action potential (SNAP) amplitude was 5 
microvolts (uv) (normal 8 uv), with normal SNCV and DL. Right calf epidermal nerve fiber (ENF) studies showed 2.4 ENF per millimeter (mm) of skin (nor$\mathrm{mal}>3.2 / \mathrm{mm}$ ) with normal ENF density in the right thigh. These findings were consistent with length-dependent large fiber symmetrical demyelinating and axonal neuropathy, and distal small fiber neuropathy.

Tests of cardiovagal parasympathetic and adrenergic sympathetic function employing a 70-degree head-up tilt for 5 minutes was performed using the WR Medical Electronics Full Autonomic $\mathrm{Lab}^{\oplus}$ that showed an immediate and sustained fall in systolic blood pressure (SBP) to a minimum of $86 \mathrm{~mm} \mathrm{Hg}$ immediately upon head-up tilting equal to a fall of $44 \mathrm{~mm} \mathrm{Hg}$ from baseline; with a non-compensatory maximal heart rate (HR) response of 89 beats per minute (bpm) reflecting a $19 \mathrm{bpm}$ change from baseline. Valsalva maneuver and HR response to deep breathing were intact. These findings were consistent with orthostatic intolerance and hypotension due to dysautonomia.

Screening metabolic, autoimmune and infectious serology were normal including serum sodium levels. Non-contrast magnetic resonance imaging (MRI) of the brain including volumetric measurements using NeuroQuant $\left(^{\circledR}\right)$ software of the hippocampus and medial temporal lobes were normal. Whole-body positron emission tomography with 2-deoxy-2-[fluorine-18] fluoro-D-glucose integrated with computed tomography $\left(\left[{ }^{18} \mathrm{~F}\right]-\mathrm{FDG} \mathrm{PET} / \mathrm{CT}\right)$ showed hypometabolism of the left temporal lobe, without evidence of systemic malignancy. Nuclear medicine (NM) cerebral perfusion with single photon emission computed tomographicbrain (SPECT) imaging with technetium-99 m showed perfusion defects in the left frontal and parietal lobes consistent with disruption of the bloodbrain barrier (BBB).

Matched serum and cerebrospinal fluid (CSF) were forwarded to the Mayo Clinic in August 2016. The serum VGKG-complex autoantibody level was 0.14 nanomoles (nM) (normal, $<=0.02 \mathrm{nM}$ ) by RIA without reactivity to LGI1 and CASPR2-IgG proteins by immunofluorescence assay (IFA) (3) and non-detectable VGKG-complex antibodies in the CSF. Serum and CSF were also negative for a panel of autoantibodies including antineuronal nuclear antibody (ANNA) types 1, 2 and 3; anti-glial/neuronal nuclear antibody type 1 (AGNA-1), Purkinje cell cytoplasmic antibody (PCA) type 1,2 and Tr; amphiphysin, and collapsin response mediator protein 5 (CRMP-5)-IgG; N-methyl-d-aspartate receptor (NMDA-R), glutamic acid decarboxylase 65 (GAD) 65; 2-amino-3-(5methyl-3-oxo-1,2-oxazol-4-yl) propanoic acid receptor (AMPA-R); N-type or $\mathrm{P} / \mathrm{Q}$-type calcium channels. CSF was acellular with normal protein, glucose, albumin and IgG levels, and absent oligoclonal bands (OCB). Bacterial, fungal, tuberculous, and viral studies, the latter employing real-time polymerase chain reaction for Adenovirus, Herpes simplex virus types 1 and 2, Cytomegalovirus, Varicella Zoster, Epstein Barr, Human Herpes virus type 6 deoxyribonucleic acid (DNA), and West Nile, Eastern Equine, and Saint Louis Encephalitis viralribonucleic acid (RNA) were all normal.

The author witnessed and recorded a brief attack commencing with disturbed 
vocalizations, facial grimacing accompanied by left head turning, neck stiffness, and left arm posturing lasting a few seconds without tonic-clonic movements, loss of consciousness, or amnesia for the event (Figure 1).

The patient was begun on 2 grams per kilogram body weight of intravenous immune globulin (IVIg).

\section{Discussion}

A patient with new-onset attacks of unilateral facial grimacing and dystonic left arm posturing that preceded neurocognitive disturbances, and associated left temporal lobe hypometabolism on [18F]-FDG imaging, and peripheral large and small fiber demyelinating and axonal neuropathy, and dysautonomia, had elevated VGKC-complex autoantibodies indicative of autoimmune limbic encephalitis (LE).

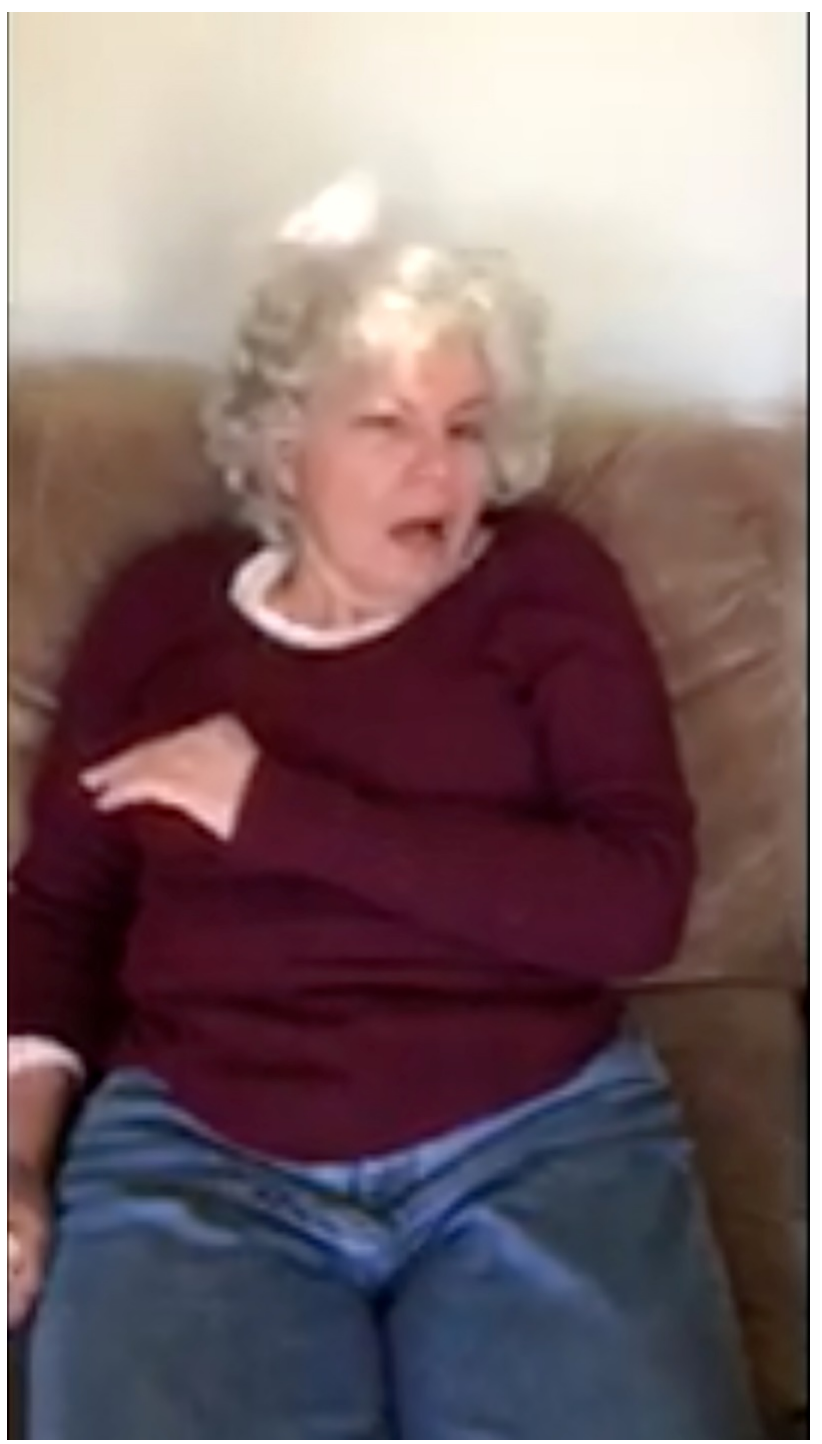

Figure 1. Faciobrachial dystonic seizure. The patient was recorded with a brief attack commencing with disturbed vocalizations, facial grimacing, left head turning and left arm posturing lasting a few seconds. 


\subsection{Historical Background}

The earliest affected patients with VGKC-complex encephalitis were described by Brierley and colleagues in 1960 [4]. Three patients, all in the seventh decade of life, similar to the present patient, presented with focal temporal lobe seizures, neuropsychiatric, and memory disturbances, including one (Case 3) with neuropathic complaints. The patients progressed from stupor and coma to death over a mean of 8 months (range 3 months to 14 months). Postmortem examination in all three showed an intense inflammatory reaction most severe in the medial parts of the temporal lobes without hemorrhage, necrosis or inclusion bodies. Although interpreted as a novel presentation of non-neoplastic subacute encephalitis, two patients were suggested to have a possible relation to cancer. Case 2 manifested had several enlarged mediastinal and hilar lymph nodes noted at postmortem examination which were infiltrated by oat-shaped cells. Chest radiograph in Case 3 disclosed a shadow in the right hilum that was negative for cancer and at postmortem found to be fibrotic lymph nodes.

In 1968, Corsellis and colleagues [5] coined the term "limbic encephalitis" noting a relation to bronchial cancer in three additional patients in the sixth to eighth decade of life, showing close clinicopathologic similarity to the cases described by Brierley and colleagues [4]. All three had subacute temporal lobe seizures, neuropsychiatric, and memory disturbances for two years before death, with resultant inflammatory lesions mainly in limbic grey matter sections of the brain, notably in medial temporal lobe structures of the uncus and amygdaloid nuclei, and in the hippocampal, cingulum and dentate gyri. In addition, Case 2 had removal of an undifferentiated non-metastatic lung carcinoma six months after onset of neurological symptoms, while two other patients had clinically unsuspected cancer at postmortem examination. Case 1 was found to have bronchial carcinoma restricted to a mediastinal lymph node without primary lesion, while Case 3 had an unsuspected oat cell carcinoma infiltrating the main bronchi of both lungs and adjacent mediastinal nodes.

By 1998, Lennon [6] and Dalmau [7] and colleagues established the paraneoplastic basis of LE in association with specific autoantibodies, including ANNA-1 $(\mathrm{Hu})$ in association with lung cancer; to MA antigen 2 (Ma2) in testicular cancer [8] and CRMP5/Cv2 in thymoma [9].

In 2000, Bien and coworkers [10] [11] described several patients with nonparaneoplastic LE without an established immunologic basis. Two patients with VGKC complex encephalitis reported by Buckley and colleagues [12] had near-complete recovery that paralleled the fall in VGKC complex IgG titers including one who was treated for recurrent thymoma and received plasma exchange (PE), and absent a detectable tumor or specific immunotherapy in the other patient. Vincent and coworkers [13] coined the term VGKC-antibody-associated "encephalopathy" to distinguish patients with potentially reversible non-paraneoplastic syndrome from those with an association to malignancy and a poorer prognosis. Irani and Vincent [14] concurred with this terminology as 
more patients were diagnosed empirically without tissue evidence of inflammation. In 2008, Tan and colleagues [15] described the clinical neurologic manifestations of VGKC-complex autoimmunity among 72 patients with non-paraneoplastic VGKC complex autoimmunity, the neurologic manifestations of which were generally subacute onset (71\%) of cognitive impairment (71\%), seizures (53\%), dysautonomia (33\%), and peripheral nerve dysfunction (25\%) similar to the present patient. In the same year, Irani and colleagues [16] described seizure-like attacks, later termed FBDS [17], for its distinctive semiology, further noting its close association with VGKC complex LGI1 antibodies and onset of LE.

\subsection{VGKC-Complex Subtyping}

The high proportion of VGKC-complex IgG-seropositive patients whose serum samples lack LGI1 IgG and CASPR2 IgG specificities, similar to the present patient, suggests that other VGKC-complex molecular targets remain to be discovered. Only about $4 \%$ to $5.5 \%$ of unselected cases were seropositive by RIA with confirmatory retesting using ${ }^{125} \mathrm{I}-\alpha$-dendrotoxin alone (radioligand for Kv1.1, Kv1.2, and Kv1.6 channels) [3] [18] [19], making the test unreliable as a screen for LE without further subtyping for LGI1 and CASPR2-IgG. So selected, 26\% [3] to $28 \%$ [19] of seropositive VGKC sera revealed reactivity with LGI1 and/or CASPR2-IgG, with a significant association between LGI1-IgG-positivity and cognitive impairment andseizures $(P<0.05)$, and CASPR2-IgG-positivity and peripheral motor excitability $(P=0.004)[3]$, however neither autoantibody was pathognomonic for a specific neurologic presentation.

Yet there was concern for screening of unselected sera for VGKC-complex antibodies by RIA. Graus and Gorman [20] argued that the VGKC-complex RIA antibody test could be used as initial screening to select positive samples that could then be confirmed by LGI1 or CASPR2-IgG antibody subtyping, however the latter were also positive in selected VGKC-complex antibody-negative sera by RIA [19] [21]. Paterson and colleagues [22] noted positive VGKC-complex antibody values ( $>400 \mathrm{pm} ;>0.4 \mathrm{nM}$ ) that were likely to be relevant in LE and related syndromes, as well as low-positive values ( $<400 \mathrm{pm} ; 0.1-0.4 \mathrm{nM})$ in 32/44 cases considered to be non-autoimmune, $4(13 \%)$ cases of which were found to have a definite or probable autoimmune disease including paraneoplastic neurologic disorder, neuromyotonia or Morvan syndrome. Ances and colleagues [23] noted that the RIA used in the clinical analysis of VGKC-complex antibodies identified a limited number of subunits (Kv1.1, Kv1.2 and Kv1.6) but that it was reasonable to speculate that antibodies to other subunits, $\mathrm{K}(+)$ channel families and VGKC ion channels might also associate with LE [13].

\subsection{Laboratory Investigation of VGKC Complex Autoimmunity}

Cerebral imaging studies in autoimmune LE show highly variable results. The present patient had temporal lobe hypometabolism on $\left[{ }^{18} \mathrm{~F}\right]$-FDG PET similar to other reported cases [24] [25] while others displayed temporal lobe hypermeta- 
bolism [26]. Baumgartner and colleagues [26] identified 9/18 (50\%) patients positive for non-paraneoplastic antibodies against neuronal surface antigens (VGKC or NMDA-R), 2 of whom displayed mesiotemporal hypermetabolism on $\left[{ }^{18} \mathrm{~F}\right]-\mathrm{FDG}$, with 4 others were rated normal, and 3 displayed hypermetabolism outside the mesiotemporal region. The fraction of abnormal scans employing MRI was lower (10/16; 62.3\%) than $\left[{ }^{18} \mathrm{~F}\right]$-FDG PET (14/18; 77.7\%). In a patient with VGKC-complex LE [25] who did not definitively demonstrate structure abnormalities on serial brain MRI over time despite ongoing temporal lobe seizures captured on video-EEG, ${ }^{18} \mathrm{~F}$-FDG fused with gadolinium-enhanced MRI nonetheless showed temporal hypometabolism.

CSF results have been equally variable in association with VGKC-complex autoimmunity. Jarius and colleagues [27] performed 29 lumbar punctures in 17 patients with VGKC-complex LE noting normal findings in up to 53\% of CSF specimens. There were no significant differences between the CSF findings and the titers of serum VGKC-complex autoantibodies. Slight pleocytosis, mainly consisting of lymphocytes and monocytes, and elevated total protein concentrations were present in $41 \%$ and $47 \%$, respectively. A disturbance of the integrity of BBB was found in 6 (35\%) patients based upon an abnormal CSF/serum humoral immune response. Absence of CSF-specific OCB, considered to be a marker of autochthonous antibody synthesis within the CNS in all patients [27] suggested an extrathecal origin of VGKC-complex autoantibodies. Vincent and colleagues [13] reported the CSF findings in 10 patients, all with VGKC-complex antibody-associated LE, noting mild lymphocytosis and mild or moderately raised protein content in one-half. OCB were noted in 1 patient, while 6 other OCB were identical to serum. VGKC-complex antibody assays on matched serum and CSF showed antibodies levels of the latter present in 4 patients that varied between $<1 \%$ and $10 \%$ of the serum, and beneath $10 \%$ in one patient with the lowest serum value. Ten positive sera retested for binding to rat brain sections revealed 5 strongly seropositive by RIA and immunohistochemistry and directed mainly against Kv1.1 and Kv1.2 subtypes of VGKC present in the molecular layer of the dentate gyrus of the hippocampus as previously shown by others [12] [28] [29]. These findings were consistent with extrathecal synthesis of VGKC-complex antibodies. It is of interest that NM brain SPECT imaging in the present patient [17] showed perfusion defects similar to 2 reported patients with FBDS and LGI1-antibody LE [17] that might explain passage of extrathecally-derived VGKC-complex antibodies across a disrupted BBB.

According to Irani and colleagues [17] 89\% of patients with FBDS were highly correlated with development of LGI1-IgG-associated LE. The attacks were typically very brief (usually $<3$ seconds) occurring up to fifty times in a day, all with arm involvement, as well as the ipsilateral face (76\%), leg (34\%) and trunk (28\%). In $69 \%$ of patients, FBDS involved either side but was always unilateral on any occasion. In $7(24 \%)$ patients, vocalizations, similar to the present patient, occurred at the start of an attack rarely with ictal loss of awareness. In a cohort of 29 affected cases [17], EEG revealed interictal diffuse or bilateral fron- 
totemporal slowing or temporal sharp waves in 17 (65\%) patients, and was normal in 9. Lumbar CSF examination was unremarkable in $20(80 \%)$, and brain MRI was normal in $12(46 \%)$, with the remaining 14 showing increased $\mathrm{T}_{2}$-signal changes in bilateral (10 patients) or unilateral temporal lobes (2 patients). The mechanism of seizures in association with LE is not well understood. Antiepileptic drugs (AED) were generally ineffective showing a reduction in FBDS frequency in only 4 (14\%) patients, often with an excess of intolerable side effects. Immunotherapy employing corticosteroids, intravenous immune globulin (IVIg), plasma exchange, or rituximab given to 26 patients was associated with an equal response in reducing attacks by $>50 \%$ or $20 \%$ to $50 \%$ in $96 \%$ of patients suggesting an autoimmune mechanism of FBDS.

Irani and Vincent [14] estimated features of peripheral neuropathy in $1.6 \%$ of VGKC-complex antibody-positive LE cases. Lahoria and coworkers [30] described 5 patients with painful polyneuropathy, all positive for VGKC-complex autoantibodies (range 0.08 to $1.18 \mathrm{nM}$ ), two of whom had antigens positive for CASPR2 and LGI1-IgG, both at low VGKC-complex antibody titers (respectively 0.08 and $0.16 \mathrm{nM} / \mathrm{L}$ ). Electrophysiological studies showed length-dependent sensorimotor polyneuropathy that was concordant with abnormal indices of axonal degeneration or demyelination in 4 nerves, and the latter with quantitative analysis of semithin sections in 2 . All 5 showed absence of inflammatory cell infiltration. Epidermal nerve fiber analysis was not performed in any of the patients in spite of painful small fiber complaints. Three patients, including one who subtyped positive for LGI1-IgG, and treated with immunotherapy had improved pain after IVIg ( 2 patients) or methotrexate ( 1 patient) combined with corticosteroids.

Neuropathic pain as a manifestation of VGKC-complex autoimmunity was noted in 316 (4\%) of 1992 patients evaluated neurologically at a tertiary referral center [31] that was typically subacute in onset, nociceptive, regional, or diffuse. In those suspected of peripheral neuropathy, the abnormality detected most commonly in EDX studies was a minor reduction of sural SNAP with motor hyperexcitability, while structured examination revealed mild subjective loss of temperature and pain attributed to small fiber dysfunction. The VGKC-complex antibody titers were often low $(0.02-0.1 \mathrm{nM})$ and antibodies to LGI1 or CASPR2 were present in $28 \%$ overall, with the latter most common (7\%). Bennett and Vincent [32] conjectured that VGKC-complex antibodies led to enhanced excitability of the somatosensory system noting that CASPR2 was an adhesion molecule that forms a complex with Kv1.1 and 1.2 in the juxtaparanodal region of the node of Ranvier capable of modulating intermodal resting potential. Its effects could also operate on the soma of C-fiber nociceptors that express VGKC currents, blockade of which could enhance neuronal excitability. Analogously, voltage-gated sodium channels were deemed responsible for the generation and conduction of action potentials in the peripheral nociceptive neuronal pathway where $\mathrm{NaV} 1.7, \mathrm{NaV} 1.8$, and $\mathrm{NaV} 1.9$ sodium channels (encoded by SCN9A, SCN10A, and SCN11A) are preferentially expressed [33]. 


\subsection{Dysautonomia}

Autonomic involvement, so noted in $29 \%$ of the cohort studied by Klein and colleagues [31] and in $3(60 \%)$ of the patients described by Lahoria and coworkers [30], was ascertained by thermoregulatory sweating or axonal reflex testing (QSART). The symptoms of small fiber neuropathy (SFN), which arise from dysfunction in nociception, temperature and autonomic modalities [34], are most adequately assessed by assessment of ENF density [35] and a combination of cardiovagal, sudomotor and adrenergic functions tests [36]. A peripheral basis for autonomic dysfunction was suspected in the present patient because of the association with SFN. Hypothermia was described in association with VGKCcomplex antibody-associated LE in 4 patients [37], Patient 1 of whom had concomitant neuropathic patient, and in the absence thereof in the others who were conjectured to have otherwise disturbed hypothalamic thermoregulatory mechanisms as the cause for dysautonomia.

\section{Treatment}

Treatment of underlying VGKC-complex-associated LE with FBDS, and concomitant distal large fiber and painful SFN, and dysautonomia, was initiated with IVIg therapy. However, the management of VGKC-complex antibody-associated LE may also include PE, and immunosuppression with corticosteroids, nonsteroidal immunosuppressive, chemotherapeutic or biological agents for refractory or more severe cases [13] [16] [38] [39]. Albeit uncommon, spontaneous remission of LE occurs in the absence of immunotherapy [24] with management alone of associated temporal lobe seizures using AED.

Bataller and colleagues [40] noted that treatment-responsiveness of LE was especially favorable among patients with antibodies to the VGKC-complex with overall improvement in two-thirds or more of patients. However, a favorable response to therapy was not limited to patients with VGKC-complex antibodies but extended to novel-cell-membrane antigens (nCMAg) expressed in the hippocampus. If the autoantigens were unknown but found to be highly enriched in neuronal cells membranes of the hippocampus, these antibodies were likewise associated with a favorable outcome emphasizing the usefulness of immunohistochemistry with PFA-fixed tissue and cultures of live hippocampus rat neurons in the analysis of highly-suspected patients with LE. The salutary effect of immunotherapy in the management of seizures in VGKC-complex antibody-associated LE supported an autoimmune basis for FBDS in the present patient and in the management of other related seizure types. Morante-Redolat [41] and Kalachikov and colleagues [42] described autosomal dominant lateral temporal epilepsy (ADLTE) characterized by partial seizures and preceding auditory signs in the LGI1/Epitempin gene expressed on chromosome 10q24. Mutations in this gene introduce premature stop codons and prevent production of full-length protein from the affected allele. Although LGI1 haploinsufficiency causes ADLTE, the underlying molecular mechanism that results in abnormal brain excitability has instead been attributed to dysregulation of synaptic AMPA recep- 
tors in hippocampal neurons in the epileptic LGI1 knock-out mouse [43]. Fukata and colleagues [44] propose that extracellularly secreted LGI1 that links two epilepsy-related brain receptors, ADAM22 and ADAM23, organizes a transsynaptic protein complex that includes presynaptic potassium channels and postsynaptic AMPA receptor scaffolds. The lack of LGI1 disrupts this synaptic protein connection and selectively reduces AMPA receptor-mediated synaptic transmission in the hippocampus.

\section{Acknowledgements}

The author expresses appreciation to Avi Gadoth MD, Mayo Clinict, Department of Neurology, Rochester, NM, for helpful comments in the preparation of the manuscript.

\section{References}

[1] Lai, M., Huijbers, M.G., Lancaster, E., et al. (2010) Investigation of LGI1 as the Antigen in Limbic Encephalitis Previously Attributed to Potassium Channels: A Case Series. The Lancet Neurology, 9, 776-785. https://doi.org/10.1016/S1474-4422(10)70137-X

[2] Irani, S.R., Alexander, S., Waters, P., et al. (2010) Antibodies to Kv1 Potassium Channel-Complex Proteins Leucine-Rich, Glioma Inactivated 1 Protein and Contactin-Associated Protein-2 in Limbic Encephalitis, Morvan's Syndrome and Acquired Neuromyotonia. Brain, 133, 2734-2748. https://doi.org/10.1093/brain/awq213

[3] Klein, C.K., Lennon, V.A., Aston, P.A., et al. (2013) Insights from LGI1 and CASPR2 Potassium Channel Complex Autoantibody Subtyping. JAMA Neurology, 70, 229-234. https://doi.org/10.1001/jamaneurol.2013.592

[4] Brierley, J.B. (1960) Subacute Encephalitis of Later Adult Life. Mainly Affecting the Limbic Areas. Brain, 83, 357-368. https://doi.org/10.1093/brain/83.3.357

[5] Corsellis, J.A., Goldberg, G.J. and Norton, A.R. (1968) “Limbic Encephalitis” Ad Its Association with Carcinoma. Brain, 91, 481-496.

https://doi.org/10.1093/brain/91.3.481

[6] Lucchinetti, C.F., Kimmel, D.W. and Lennon, V.A. (1998) Paraneoplastic and Oncologic Profiles of Patients Seropositive for Type 1 Antineuronal Nuclear Autoantibodies. Neurology, 50, 652-657. https://doi.org/10.1212/WNL.50.3.652

[7] Dalmau, J., Graus, F., Rosenblum, M.K., et al. (1992) Anti-Hu-Associated Paraneoplastic Encephalomyelitis/Sensory Neuronopathy. A Clinical Study of $71 \mathrm{~Pa}-$ tients. Medicine (Baltimore), 71, 59-72. https://doi.org/10.1097/00005792-199203000-00001

[8] Voltz, R., Gultekin, S.H., Rosenfeld, M.R., et al. (1999) A Serological Marker of Paraneoplastic Limbic and Brain-Stem Encephalitis in Patients with Testicular Cancer. The New England Journal of Medicine, 340, 1788-1795.

https://doi.org/10.1056/NEJM199906103402303

[9] Antoine, J.C., Honnorat, J., Anterion, C.T., et al. (1995) Limbic Encephalitis and Immunological Perturbations in Two Patients with Thymoma. Journal of Neurology, Neurosurgery \& Psychiatry, 58, 706-710. https://doi.org/10.1136/jnnp.58.6.706

[10] Bien, C.G., Schulze-Bonhage, A., Deckert, M., et al. (2000) Limbic Encephalitis Not Associated with Neoplasm as a Cause of Temporal Lobe Epilepsy. Neurology, 55, 1823-1828. https://doi.org/10.1212/WNL.55.12.1823 
[11] Mori, M., Kuwabara, S., Yoshiyama, M., et al. (2002) Successful Immune Treatment for Non-Paraneoplastic Limbic Encephalitis. Journal of the Neurological Sciences, 201, 85-88. https://doi.org/10.1016/S0022-510X(02)00188-0

[12] Buckley, C., Oger, J., Clover, L., et al. (2001) Potassium Channel Antibodies in Two Patients with Reversible Limbic Encephalitis. Annals of Neurology, 50, 73-78. https://doi.org/10.1002/ana.1097

[13] Vincent, A., Buckley, C., Schott, J.M., et al. (2004) Potassium Channel AntibodyAssociated Encephalopathy: A Potentially Immunotherapy-Responsive Form of Limbic Encephalitis. Brain, 127, 701-712. https://doi.org/10.1093/brain/awh077

[14] Irani, S.R. and Vincent, A. (2016) Voltage-Gated Potassium Channel-Complex Autoimmunity and Associated Clinical Syndrome. Handbook of Clinical Neurology, 133, 185-197. https://doi.org/10.1016/B978-0-444-63432-0.00011-6

[15] Tan, K.M., Lennon, V.A., Klein, C.J., et al. (2008) Clinical Spectrum of VoltageGated Potassium Channel Autoimmunity. Neurology, 70, 1893-1890. https://doi.org/10.1212/01.wnl.0000312275.04260.a0

[16] Irani, S.R., Buckley, C., Vincent, A., et al. (2008) Immunotherapy-Responsive Seizure-Like Episodes with Potassium Channel Antibodies. Neurology, 71, 1647-1648. https://doi.org/10.1212/01.wnl.0000326572.93762.51

[17] Irani, S.R., Michell, A.W., Lang, B., et al. (2011) Faciobrachial Dystonic Seizures Precede Lgil Antibody Limbic Encephalitis. Annals of Neurology, 69, 892-900. https://doi.org/10.1002/ana.22307

[18] Apiwattanakul, M., McKeon, A., Pittock, S.J., et al. (2010) Eliminating False-Positive Results in Serum Tests for Neuromuscular Autoimmunity. Muscle \& Nerve, 41, 702-704. https://doi.org/10.1002/mus.21653

[19] Van Sonderen, A., Schreurs, M.W., de Bruijn, M.A., et al. (2016) The Relevance of VGKC Positivity in the Absence of LGI1 and Caspr2 Antibodies. Neurology, 86, 1692-1699. https://doi.org/10.1212/WNL.0000000000002637

[20] Graus, F. and Gorman, M.P. (2016) Voltage-Gated Potassium Channel Antibodies. Neurology, 86, 1657-1658. https://doi.org/10.1212/WNL.0000000000002644

[21] Irani, S.R., Pettingill, P., Kleopa, K.A., et al. (2012) Morvan Syndrome: Clinical and Serological Observations in 29 Cases. Annals of Neurology, 72, 241-255.

https://doi.org/10.1002/ana.23577

[22] Paterson, R.W., Zandi, M.S., Armstrong, R., Vincent, A. and Schott, J.M. (2014) Clinical Relevance of Positive Voltage-Gated Potassium Channel (VGKC)-Complex Antibodies: Experience from a Tertiary Referral Centre. Journal of Neurology, Neurosurgery \& Psychiatry, 85, 625-630. https://doi.org/10.1136/jnnp-2013-305218

[23] Ances, B.M., Vitaliani, R., Taylor, R.A., et al. (2005) Treatment-Responsive Limbic Encephalitis Identified by Neuropil Antibodies: MRI and PET Correlates. Brain, 128, 1764-1777. https://doi.org/10.1093/brain/awh526

[24] Gast, H., Schindler, K., Z'Graggen, W.J. and Hess, C.W. (2010) Improvement of Non-Paraneoplastic Voltage-Gated Potassium Channel Antibody-Associated Limbic Encephalitis without Immunosuppressive Therapy. Epilepsy \& Behavior, 17, 555-557. https://doi.org/10.1016/j.yebeh.2010.01.017

[25] Day, B.K., Eisenman, L., Black, J., Maccotta, L. and Edward Hogan, R. (2015) A Case Study of Voltage-Gated Potassium Channel Antibody-Related Limbic Encephalitis with PET/MRI Findings. Epilepsy \& Behavior Case Reports, 4, 23-26. https://doi.org/10.1016/j.ebcr.2015.02.002

[26] Baumbartner, A., Rauer, S., Mader, I. and Meyer, P.T. (2013) Cerebral FDG-PET and MRI Findings in Autoimmune Limbic Encephalitis: Correlation with Autoan- 
tibody Types. Journal of Neurology, 260, 2744-2753.

https://doi.org/10.1007/s00415-013-7048-2

[27] Jarius, S., Hoffman, L., Clover, L., et al. (2008) CSF Findings in Patients with Voltage Gated Potassium Channel Antibody Associated Limbic Encephalitis. Journal of the Neurological Sciences, 268, 74-77. https://doi.org/10.1016/j.jns.2007.11.004

[28] Rhodes, K.J., Monagham, M.M., Barrezueta, N.X., et al. (1996) Voltage-Gated K+ Channel Beta Subunits: Expression and Distribution of Kvbeta1 and Kvbeta2 in Adult Rat Brain. Journal of Neuroscience, 16, 4846-4860.

[29] Monaghan, M.M., Trimmer, J.S. and Rhodes, K.J. (2001) Experimental Localization of Kv1 Family Voltage-Gated K+ Channel Alpha and Beta Subunits in Rat Hippocampal Formation. Journal of Neuroscience, 21, 5973-5983.

[30] Lahoria, R., Pittock, S.J., Gadoth, A., et al. (2016) Clinical-Pathologic Correlations in VGKC-Subtyped Autoimmune Painful Polyneuropathy. Muscle \& Nerve, Epub Ahead of Print. https://doi.org/10.1002/mus.25371

[31] Klein, C.J., Lennon, V.A., Aston, P.A., McKeon, A. and Pittock, S.J. (2012) Chronic Pain as a Manifestation of Potassium Channel-Complex Autoimmunity. Neurology, 79, 1136-1144. https://doi.org/10.1212/WNL.0b013e3182698cab

[32] Bennett, D.L.H. and Vincent, A. (2012) Autoimmune Pain. Neurology, 79, 1080 1081. https://doi.org/10.1212/WNL.0b013e3182698dc3

[33] Brouwer, B.A., Merkies, I.S., Gerrits, M.M., et al. (2014) Painful Neuropathies: The Emerging Role of Sodium Channelopathies. Journal of the Peripheral Nervous System, 19, 53-65. https://doi.org/10.1111/jns5.12071

[34] Chan, A.C. and Wilder-Smith, E.P. (2016) Small Fiber Neuropathy: Getting Bigger! Muscle \& Nerve, 53, 671-682. https://doi.org/10.1002/mus.25082

[35] Lacomis, D. (2002) Small-Fiber Neuropathy. Muscle \& Nerve, 26, 173-188. https://doi.org/10.1002/mus.10181

[36] Low, P.A., Tomalia, V.A. and Park, K.J. (2013) Autonomic Function Tests: Some Clinical Applications. Journal of Clinical Neurology, 9, 1-8. https://doi.org/10.3988/jcn.2013.9.1.1

[37] Jacob, S., Irani, S.R., Rajabally, A., et al. (2008) Hypothermia in VGKC Antibody-Associated Limbic Encephalitis. Journal of Neurology, Neurosurgery \& Psychiatry, 79, 202-204. https://doi.org/10.1136/jnnp.2007.130039

[38] Irani, S.R., Gelfand, J.M., Bettcher, B.M., Singhal, N.S. and Geschwind, M.D. (2014) Effect of Rituximab in Patients with Leucine-Rich, Glioma-Inactivated 1 AntibodyAssociated Encephalopathy. JAMA Neurology, 71, 896-900.

https://doi.org/10.1001/jamaneurol.2014.463

[39] Shin, Y.W., Lee, S.T., Shin, J.W., et al. (2013) VGKC-Complex/LGI1-Antibody Encephalitis: Clinical Manifestations and Response to Immunotherapy. Journal of Neuroimmunology, 265, 75-81. https://doi.org/10.1016/j.jneuroim.2013.10.005

[40] Bataller, L., Kleopa, K.A., Wu, G.F., et al. (2007) Autoimmune Limbic Encephalitis in 39 Patients: Immunophenotypes and Outcomes. Journal of Neurology, Neurosurgery \& Psychiatry, 78, 381-385. https://doi.org/10.1136/jnnp.2006.100644

[41] Morante-Redolat, J.M., Gorostidi-Pagola, A., Piquer-Sirerol, S., et al. (2002) Mutations in the LGI1/Epitemprin Gene on 10q24 Cause Autosomal Dominant Lateral Temporal Epilepsy. Human Molecular Genetics, 11, 1119-1128. https://doi.org/10.1093/hmg/11.9.1119

[42] Kalachikov, S., Evgrafov, O., Ross, B., et al. (2002) Mutations in LGI1 Cause Autosomal-Dominant Partial Epilepsy with Auditory Features. Nature Genetics, 30, 335 - 
341. https://doi.org/10.1038/ng832

[43] Ohkawa, T., Fukata, Y., Yamasaki, M., et al. (2013) Autoantibodies to Epilepsy-Related LGI1 in Limbic Encephalitis Neutralize LGI1-ADAM22 Interaction and Reduce Synaptic AMPA Receptor. Journal of Neuroscience, 33, 18161-18174. https://doi.org/10.1523/JNEUROSCI.3506-13.2013

[44] Fukata, Y., Lovero, K.L., Iwanaga, T., et al. (2010) Disruption of LGI1-Linked Synaptic Complex Causes Synaptic Transmission and Epilepsy. Proceedings of the National Academy of Sciences of the United States of America, 107, 3799-3804. https://doi.org/10.1073/pnas.0914537107

\section{Scientific Research Publishing}

Submit or recommend next manuscript to SCIRP and we will provide best service for you:

Accepting pre-submission inquiries through Email, Facebook, LinkedIn, Twitter, etc. A wide selection of journals (inclusive of 9 subjects, more than 200 journals)

Providing 24-hour high-quality service

User-friendly online submission system

Fair and swift peer-review system

Efficient typesetting and proofreading procedure

Display of the result of downloads and visits, as well as the number of cited articles Maximum dissemination of your research work

Submit your manuscript at: http://papersubmission.scirp.org/

Or contactwjns@scirp.org 